\title{
Use of a novel herbal medicine in a 75-year-old woman with multi-metastatic pancreatic cancer: A case report and review of the literature
}

\author{
YANCHU LI ${ }^{1}$, XIANYONG LI ${ }^{2}$, PAIROTETORSAK TIP ${ }^{3}$ and LINGYAN ZHANG ${ }^{2}$ \\ ${ }^{1}$ Oncology Department, Western China Hospital of Sichuan University, Chengdu, Sichuan 610041; \\ ${ }^{2}$ Department of Oncology, Chengdu Fuxing Hospital, Chengdu, Sichuan 610036, \\ P.R. China; ${ }^{3}$ Department of Functional Medicine, Better Being Hospital, Bangkok 10110, Thailand
}

Received August 15, 2014; Accepted April 24, 2015

DOI: $10.3892 / \mathrm{ol} .2015 .3237$

\begin{abstract}
Pancreatic cancer is one of the most aggressive types of malignant tumors and is associated with an extremely poor prognosis. Despite numerous research efforts over the last few years, little progress has been made in the understanding and treatment of the disease. Gemcitabine-based regimens are considered as the first-line treatment for pancreatic cancer, but the effects of chemotherapy on the disease are limited. Natural products extracted from herbs represent a valuable resource for novel bioactive anticancer agents and could benefit multi-metastasis pancreatic adenocarcinoma patients with an Eastern Cooperative Oncology Group status of 3. Biological intra-control cancer treatment (BICT) is a novel systemic therapy involving palliative care and herbal extract combinations [including ginseng (Panax ginseng C.A. Mey.), Herba Agrimonia (Agrimonia pilosa Ledeb.), White Flower Patrinia Herb (Thlaspi arvense Linn.) and arginine], which has been approved by the State Food and Drug Association. The treatment is intended to regulate and inhibit blood vessel generation and tumor growth by inhibiting epidermal growth factor receptor and vascular endothelial growth factor receptor expression, and to manage symptoms to improve the quality of the treatment. The present study discusses the case of a 75-year-old female diagnosed with pancreatic cancer with multiple metastases in the liver and lymph nodes. The patient was administered BICT and achieved survival for 11 months without side-effects of a severity greater than grade 1 according to the Common Terminology Criteria for Adverse Events. The study also describes a possible approach to providing
\end{abstract}

Correspondence to: Dr Yanchu Li, Oncology Department, Western China Hospital of Sichuan University, 37 Guoxu Xiang, Wuhou, Chengdu, Sichuan 610041, P.R. China

E-mail: lyc328@yeah.net

Key words: metastasis, pancreatic cancer, herbal medicine, elderly patient, prognosis palliative care and treating late-stage, metastatic pancreatic adenocarcinomas in elderly patients.

\section{Introduction}

Pancreatic cancer is one of the most aggressive and lethal malignant tumors, but despite this, is currently lacking in efficient treatment options (1). Among patients with metastatic disease, the median survival time is $\sim 6$ months, with a 5-year survival rate of only $2 \%$, and a 1 -year survival rate of $17-23 \%$ reported following gemcitabine treatment (2). First-line chemotherapy drugs approved for the treatment of pancreatic cancer are gemcitabine and 5-fluorouracil, but gemcitabine in combination with nab-paclitaxel or erlotinib has also been approved and has been demonstrated to improve the progression-free survival (PFS) and overall survival (OS) rates compared with those associated with gemcitabine alone, together with acceptable side-effects (3). Treatment success in advanced pancreatic cancer is based on the cancer disappearing or remaining stable, but importantly, other factors such as the Eastern Cooperative Oncology Group (ECOG) (4) performance status and any potential side-effects must also be considered, particularly for combination agents.

However, the majority of patients with advanced or metastatic pancreatic cancer are elderly individuals with an ECOG performance status of 2-3, who cannot tolerate the side-effects of chemotherapy. Thus, advanced pancreatic cancer is a model illness to study low toxicity anticancer treatments and supportive management strategies (5). Palliative treatment is currently the only beneficial treatment for end-stage, metastatic pancreatic cancer. At the American Society of Clinical Oncology (ASCO) annual meeting in 2013, Rodriguez et al and Burke et al reported that only $4.2 \%$ of end-stage metastatic pancreatic cancer patients received chemotherapy at the MD Anderson Cancer Center (USA) between 2010 and 2012, and that 98.6\% of these patients only received standard agents $(6,7)$. However, even if a patient responds to treatment with a gemcitabine-based agent, this has not been demonstrated to improve the quality of life (QOL) significantly, and there is no evidence supporting a favorable outcome associated with chemotherapy in elderly patients with metastatic pancreatic cancer (8). 
In recent years, improved symptom control in patients with end-stage cancer by palliative care services has been provided with a lot of attention. On one hand, palliative treatment is capable of improving body mass index and QOL significantly, which is associated with the OS time $(9,10)$, but on the other hand, it is worth questioning what alternatives to palliative care are available, when chemotherapy or other treatments associated with severe side-effects are not an option. Therefore, the development of effective anticancer compounds with low toxicity is critical for unresectable, advanced pancreatic cancer that cannot be treated by chemotherapy. As previously described, targeted therapies aimed at vascular endothelial growth factor receptor (VEGFR), epidermal growth factor receptor (EGFR), cyclooxygenase-2, mammalian target of rapamycin, cell cycle check-points or proteasomes have been studied and are associated with a relatively low toxicity (3).

The current study presents a novel angle on understanding cancer and reports on the use of a novel systemic therapy known as biological intra-control cancer treatment (BICT), provided by Chengdu Fuxing Hospital (Chengdu, Sichuan, China) and approved by the State Food and Drug Association (SFDA). This treatment involves early palliative care and the herbal extract combinations of ginseng (Panax ginseng C.A. Mey.), and White Flower Patrinia Herb (Thlaspi arvense Linn.), Herba Agrimonia (Agrimonia pilosa Ledeb.) and arginine (WHA), among others, which are associated with low toxicity and have been approved by the SFDA. BICT is intended to regulate interactive signals between cells and to improve the QOL of patients. Our group has recently found that BICT inhibits EGFR and VEGFR expression, promotes apoptosis (unpublished data) and blocks the cell cycle in the $\mathrm{S}$ phase (11). The present study describes a novel herbal treatment used to improve survival time and QOL in a terminal pancreatic cancer patient. Written informed consent was obtained from the patient's family.

\section{Case report}

A 75-year-old female was diagnosed with pathologically confirmed multi-metastatic pancreatic cancer (liver metastatic adenocarcinoma, compatible with primary pancreatic cancer), and presented with discomfort in the liver area and weight loss (5 kg in 1 month) on November 23, 2012 at St. Anthony Hospital North (Lakewood, CO, USA). The cancer was diagnosed as American Joint Committee on Cancer stage IV pancreatic cancer (12), and the estimated survival time was $<3$ months. A physical examination revealed upper right quadrant tenderness without jaundice. A large solid and irregular mass could be palpated in the upper right quadrant. The laboratory examination findings were as follows: Serum liver enzyme levels were elevated [alanine aminotransferase, $11 \mathrm{U} / 1$ (normal range, 0-40 U/1); aspartate aminotransferase, $18 \mathrm{U} / 1$ (normal range, 0-49 U/1); alkaline phosphatase, $92 \mathrm{U} / 1$ (normal range, 34-114 U/1); and $\gamma$-glutamyl transferase, $50 \mathrm{U} / 1$ (normal range, 11-49 U/1)]; the total bilirubin level was $7.4 \mu \mathrm{mol} / 1$ (normal range, 3.0-20.0 $\mu \mathrm{mol} / \mathrm{l}$ ); the international normalized ratio and albumin level were normal; the serum tumor marker cancer antigen (CA)19-9 level was $81.42 \mathrm{U} / \mathrm{ml}$ (normal range, 0-27 U/ml); and the carcinoembryonic antigen level was normal. Computed tomography (CT) scans showed a large solid mass, $3.1 \times 4.7 \times 6.1 \mathrm{~cm}$ in size, near the pancreatic head and multiple masses in the liver, with the largest mass measuring $\sim 6.0 \times 7.0 \mathrm{~cm}$ and being located in the inferior right hepatic lobe. The right lobe of the liver appeared to be nearly completely replaced by the neoplasm. Intra- and extra-hepatic bile duct dilatations were also observed.

After recovering from a stent insertion on December 16, 2012, a baseline CT scan was performed and BICT was initiated (Fig. 1A). At this time, the liver masses were still expanding, since the pancreatic cancer was diagnosed prior to the initiation of BICT. In comparison to the results on November 26, 2012, the CT scan revealed that the largest solid mass in the region of the inferior right hepatic lobe was $\sim 6.0 \times 10.9 \mathrm{~cm}$, as compared to $\sim 6.0 \times 7.0 \mathrm{~cm}$, and the smaller mass in the liver had increased from $2.4 \times 2.7$ to $3.1 \times 3.9 \mathrm{~cm}$ in one month. The patient refused to undergo chemotherapy due to an ECOG performance status of 3, and a survival time of $<3$ months was estimated by the oncologist. The WHA herbal extract combination was orally administered as a $3.0-\mathrm{g}$ capsule plus $15 \mathrm{ml}$ in liquid form four times daily for a total of 57 days. Furthermore, hyperthermia was induced in the upper abdominal area to promote drug absorption, and abdominal palpation was performed every week to evaluate the size of the liver and to assess the risk of bleeding. Bleeding was not noted, and after two months of treatment, on February 11, 2013, an abdominal $\mathrm{CT}$ scan revealed a stable pancreatic lesion and no new metastatic lesions (Fig. 1B). According to the Response Evaluation Criteria in Solid Tumors (RECIST), the liver disease was under control (stable disease) with no discomfort or pain in the abdomen (13). Tumor marker CA19-9 and bilirubin levels were normal, and the clinical improvement was confirmed by imaging tests.

Between March and June 2013, the patient did not receive BICT due to drug shipment issues between China and the United States. CT was performed again 4 months after the initiation of treatment, on May 24, 2013, and revealed that the large lesion had not changed in size, but that new small metastatic masses had developed (Fig. 2A). From June 23, 2013, the patient received functional medicinal treatment, a type of palliative treatment, in Bangkok, and BICT was reinitiated. This resulted in clinical and QOL improvements. The tumor marker levels were restored to normal and the tumor masses exhibited a stable disease state (Fig. 2B). The patient has remained alive for 11 months since the initial diagnosis.

\section{Discussion}

Pancreatic cancer can be classified as resectable or unresectable. The prognosis is much better for resectable pancreatic cancer than for unresectable cancer cases. Surgery is considered as the only potential cure for the disease, and the mean 5-year survival rate increases to $>20 \%$ in those who have undergone a resection as part of their multimodal therapy (14-16). Furthermore, complete tumor resection, in addition to tumor-specific characteristics, including CA19-9 concentration, tumor size and differentiation, lymph node involvement, and perineural infiltration or lymphovascular invasion, are likely to be the prognostic factors that are the most relevant for patients with resectable pancreatic cancer (15). Adjuvant treatment following surgery has been has been associated with a reduced risk of 

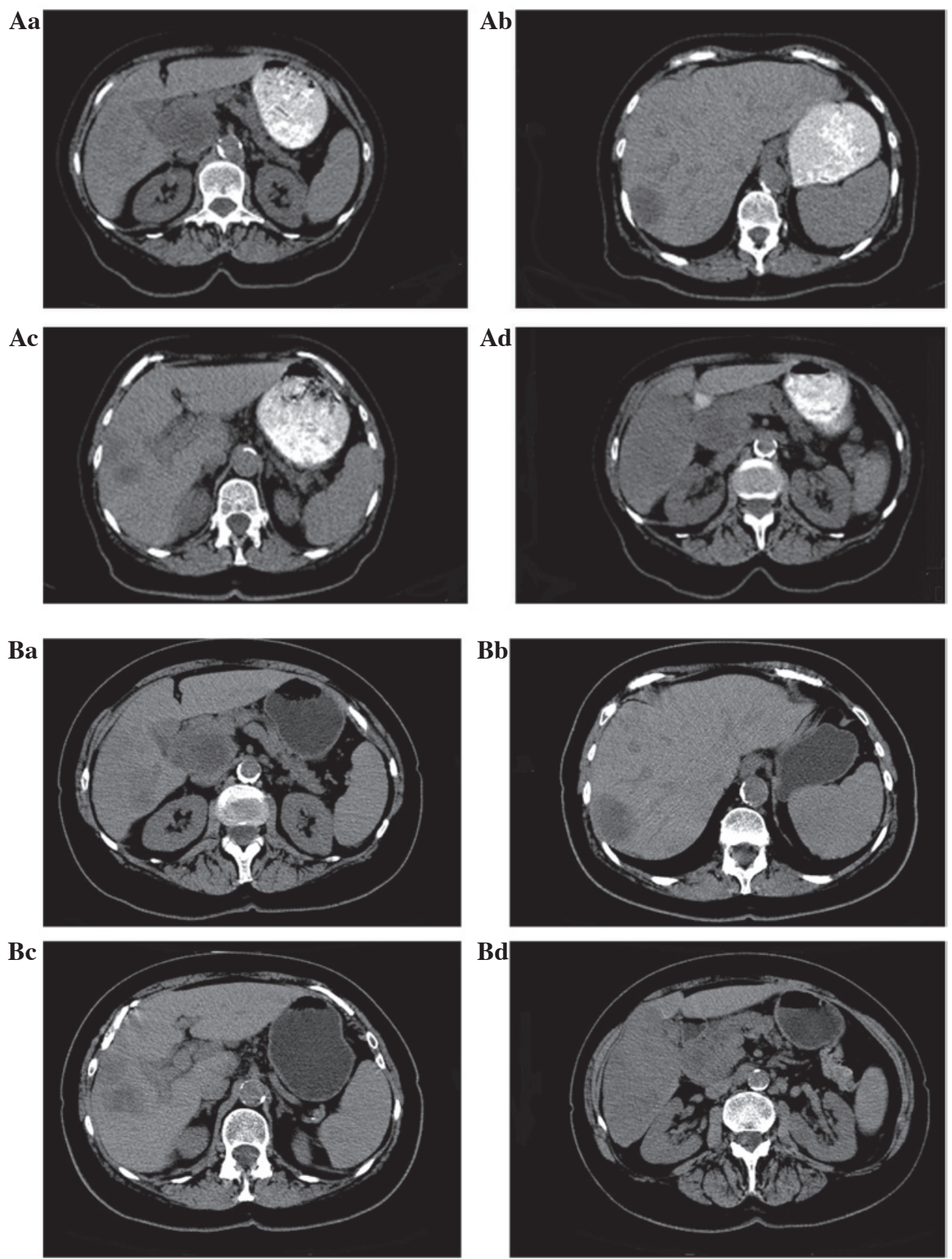

Figure 1. Evaluation of treatment by computed tomography (CT) scan following the first course treatment. No new metastatic masses were found on February 11 , 2013, subsequent to 2 months of treatment with biological intra-control cancer treatment. CT scans revealed that compared with the (A) 6.0x10.9-cm mass on December 16, 2012, the largest solid mass in the region of the inferior right hepatic lobe was now (B) $6.0 \mathrm{x} 11.2 \mathrm{~cm}$. All other masses in the right lobe of the liver remained stable.

relapse and extended survival, due to the high recurrence risk, as demonstrated by the European Study Group of Pancreatic Cancer (ESPAC) in the ESPAC-1 and ESPAC-3 trials (17). The CONKO-001 and Asian JSAP-02 trials have furthermore confirmed that surgery in addition to adjuvant chemotherapy is associated with increased disease-free survival, median survival and 5-year OS rates compared with surgery alone $(16,18,19)$. The major adjuvant treatments after resection of pancreatic cancer traditionally include chemotherapy, fluorouracil-based chemo-radiation, and chemo-radiation plus chemotherapy. However, the optimum treatment remains controversial. Evidently, there is a definite requirement for the development of alternative agents with novel mechanisms of action to treat this disease. The reality is that a number of patients, and particularly elderly patients, suffer from advanced or metastatic pancreatic cancer that is unresectable. Liver metastasis is an independent adverse predictor of survival, and for patients with unresectable pancreatic cancer, liver metastasis is associated with an extremely poor prognosis. Even if patients can tolerate chemotherapy, the average OS time is $\sim 9$ months according to one previous study (20). A recent clinical trial demonstrated that the median OS time could be extended to 8.5 months in patients treated with nab-paclitaxel-gemcitabine, as compared with 6.7 months in patients treated with gemcitabine alone (2), suggesting that patients may benefit from combination regimens. Hence, if the patients have the opportunity to undergo 

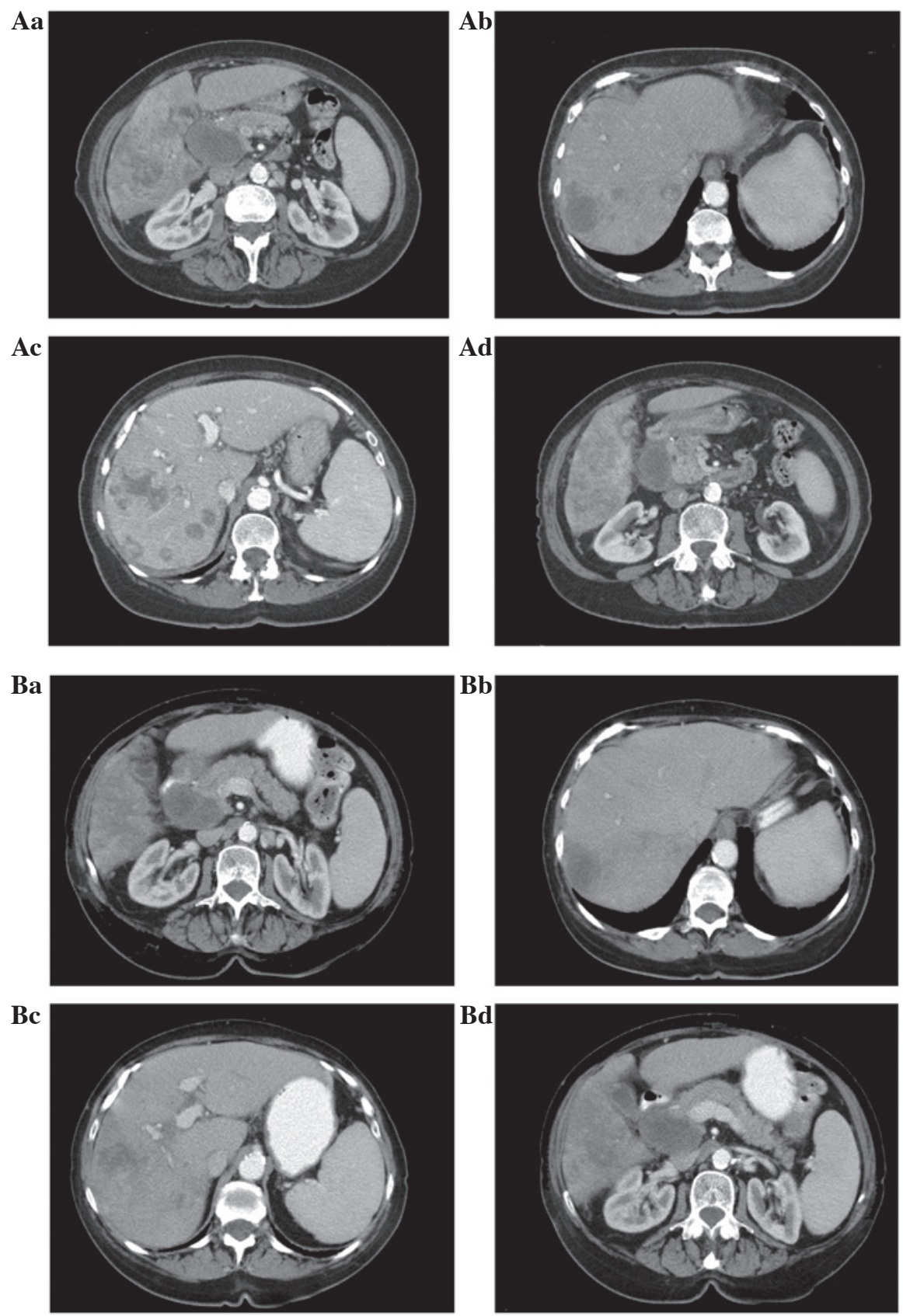

Figure 2. Evaluation of treatment by computed tomography (CT) scan following the reinitiated treatment. (A) On May 24, 2013, CT scans showed large solid masses near the head of the pancreas, and this was accompanied by new masses in the liver, as compared to February 11, 2013. (B) No new metastases were found since the biological intra-control cancer treatment was renewed, and CT scans revealed that the large solid mass in the inferior right hepatic lobe was $\sim 6.0 \times 11.2 \mathrm{~cm}$ on July $25,2013$.

surgery, or are able to tolerate chemotherapy, this is an effective strategy.

One of the most difficult and challenging barriers to overcome is how to deal with elderly, end-stage pancreatic cancer patients, particularly those who cannot tolerate chemotherapy and have a life expectancy of $<3$ months. Few studies have addressed this, partly since chemotherapy will severely affect the QOL of patients in this group $(21,22)$. For this reason, and since cancer is not just a mass, but rather a syndrome associated with systemic comorbidities that negatively impact the QOL and overall health of the patient, palliative treatment has become a focus of attention (23). Palliative care focuses on managing symptoms and on psychosocial support.
Temel et al (24) demonstrated that early palliative care can improve QOL and prolong survival in patients with metastatic cancer compared with standard care alone. In recent years, cancer has been considered a systemic disease. According to the RECIST, evaluation criteria only focus on the tumor mass size and not on the entire body, and consider factors such as OS or PFS that are only indicative of the tumor size and not QOL. For advanced pancreatic cancer, not only is it difficult to control the pancreatic cancer mass itself, but the QOL and OS generally cannot be improved significantly as well. As aforementioned, in the 2013 ASCO meeting, the current methods of treating advanced pancreatic cancer were questioned and it was concluded that chemotherapy may not always be the only 
answer. For patients classified as having an ECOG performance status of 3-4, no standard chemotherapy regimen has been established owing to the associated high toxicity and low effectiveness of these drugs. For these patients, individual care is commonly employed (18).

Thus, if possible, in addition to supportive and palliative treatments, patients should receive low toxicity anticancer treatments to control the cancer, as a novel strategy to prolong life. Novel treatment strategies based on this idea are currently being developed in laboratories and clinics worldwide. One of these novel treatments is BICT; a systemic therapy comprising palliative care with herbal extract combinations of $P$. ginseng and WHA, among others, which could inhibit cancer growth and improve symptoms. Although the cell toxicity of BICT is not as strong as that of gemcitabine-based combinations, it remains effective, while being much less toxic. Herbal extract combinations such as WHA have previously been demonstrated to be effective against colon and liver cancer in vitro and in vivo, and have been demonstrated to possess anti-angiogenic characteristics. We have recently shown that EGFR and VEGFR expression can be inhibited and that apoptosis can occur in vivo in breast cancer and rectal cancer models (unpublished data).

In summary, the patient described in the present case study was a 75-year-old female with end-stage pancreatic cancer and multiple liver metastases, with a predicted survival time of only 3 months. Due to the patient's advanced age and the late stage of the disease, surgery and chemotherapy were not suitable treatment options. For these types of patients, the goals of treatment are to decrease or control tumor burden, in order to reduce the risk of jaundice and bleeding in the liver, which are independent prognostic factors. The use of BICT enabled the achievement of this goal, and the patient has thus far survived for 11 months post-diagnosis. Furthermore, according to the Common Terminology Criteria for Adverse Events, version 3.0, no vomiting or hematological toxicity of more than grade 1 severity was observed (25). During the entire treatment period, only ibuprofen was used as an analgesic. Oral BICT is administered daily and can be administered at home. Hence, this treatment is not limited by the requirement for travel or ambulation, resulting in high levels of compliance. In the present case, BICT was successful in improving the QOL and prolonged the patient's survival time.

This study describes a novel approach for treating advanced metastatic pancreatic cancer, and supports the use of BICT in patients with an ECOG performance status of 2/3, thereby enabling the prolongation of survival in patients who cannot tolerate chemotherapy and resulting in an improvement in their QOL. Further research should be performed prior to conclusions being formed with regard to the mechanisms underlying BICT and the associated benefits.

\section{References}

1. Breuer S, Maimon O, Appelbaum L, et al: TL-118-anti-angiogenic treatment in pancreatic cancer: a case report. Med Oncol 30: 585, 2013

2. Von Hoff DD, Ervin T, Arena FP, et al: Increased survival in pancreatic cancer with nab-paclitaxel plus gemcitabine. N Engl J Med 369: 1691-1703, 2013.

3. Zagouri F, Sergentanis TN, Chrysikos D, et al: Molecularly targeted therapies in metastatic pancreatic cancer: a systematic review. Pancreas 42: 760-773, 2013.
4. Oken MM, Creech RH, Tormey DC, et al: Toxicity and response criteria of the Eastern Cooperative Oncology Group. Am J Clin Oncol 5: 649-655, 1982

5. Shahrokni A and Saif MW: Metastatic pancreatic cancer: The dilemma of quality vs. quantity of life. JOP 14: 391-394, 2013.

6. Rodriguez MA, DeJesus YA, Cheng L, Buzdar A and Burke TW: Factors related to end-of-life (EOL) chemotherapy in solid tumor (ST) patients. J Clin Oncol 31: abstract 9538, 2013.

7. Burke T, DeJesus Y, Cheng L, Buzdar A and MA R: Pattern of chemotherapy use at end-of-life (EOL) in patients with solid tumors (ST). J Clin Oncol 31: abstract 9539, 2013.

8. Romanus D, Kindler HL, Archer L, et al: Does health-related quality of life improve for advanced pancreatic cancer patients who respond to gemcitabine? Analysis of a randomized phase III trial of the cancer and leukemia group B. J Pain Symptom Manage 43: 205-217, 2012.

9. Li D, Morris JS, Liu J, Hassan MM, Day RS, Bondy ML and Abbruzzese JL: Body mass index and risk, age of onset and survival in patients with pancreatic cancer. JAMA 301: 2553-2562, 2009

10. Greer JA, Pirl WF, Jackson VA, et al: Effect of early palliative care on chemotherapy use and end-of-life care in patients with metastatic non-small-cell lung cancer. J Clin Oncol 30: 394-400, 2012.

11. Li X, Zhang L and Li Y: Inhibition of cancer cell proliferation by color modulation: A pilot study. Ann Oncol 24 (Suppl 9): ix83, 2013.

12. Edge S, Byrd DR, Compton CC, Fritz AG, Greene FL and Trotti A: AJCC cancer staging handbook. In: AJCC Cancer Staging Manual. 7th edition. Springer, New York, pp63-79, 2010.

13. Eisenhauer EA, Therasse P, Bogaerts J, Schwartz LH, Sargent D, et al: New response evaluation criteria in solid tumours: Revised RECIST guideline (version 1.1). Eur J Cancer 45: 228-247, 2009

14. Neoptolemos JP, Stocken DD, Bassi C, et al: Adjuvant chemotherapy with fluorouracil plus folinic acid vs gemcitabine following pancreatic cancer resection: a randomized controlled trial. JAMA 304: 1073-1081, 2010

15. Hartwig W, Werner J, Jäger D, et al: Improvement of surgical results for pancreatic cancer. Lancet Oncol 14: e476-e485, 2013.

16. Helmut Oettle SP, Peter Neuhaus, Klaus Gellert, et al: Adjuvant chemotherapy with gemcitabine vs observation in patients undergoing curative: intent resection of pancreatic cancer - a randomized controlled trial. JAMA 297: 267-277, 2007.

17. Neoptolemos JP, Stocken DD, Tudur Smith C, et al: Adjuvant 5 -fluorouracil and folinic acid vs observation for pancreatic cancer: composite data from the ESPAC-1 and -3(v1) trials. Br J Cancer 100: 246-250, 2009.

18. Oettle H, Neuhaus P, Hochhaus A, et al: Adjuvant chemotherapy with gemcitabine and long-term outcomes among patients with resected pancreatic cancer: the CONKO-001 randomized trial. JAMA 310: 1473-1481, 2013

19. Ueno H, Kosuge T, Matsuyama Y, et al: A randomised phase III trial comparing gemcitabine with surgery-only in patients with resected pancreatic cancer: Japanese study group of adjuvant therapy for pancreatic cancer. Br J Cancer 101: 908-915, 2009.

20. Niu L, Chen J, He L, Liao M, Yuan Y, Zeng J, Li J, Zuo J and $\mathrm{Xu} \mathrm{K}$ : Combination treatment with comprehensive cryoablation and immunotherapy in metastatic pancreatic cancer. Pancreas 21: 1143-1149, 2013.

21. Epstein AS, Abou-Alfa GK, Shamseddine A, Al-Olayan A, Ang C, Naghy M, Lowery MA and O'Reilly EM: Communication and palliative care in a 64-year-old man with pancreatic adenocarcinoma. Gastrointest Cancer Res 5: 130-134, 2012.

22. Panagiotarakou M, Gupta A, Syrigos K and Saif MW: Use of supportive care for symptom management in pancreatic cancer: application of clinical research to patient care. JOP 13: 342-344, 2012.

23. Paul E and Kenneth P Olive: Pancreatic cancer: why is it so hard to treat? Ther Adv Gastroenterol 6: 321-337, 2013.

24. Temel JS, Greer JA, Muzikansky A, Gallagher ER, Admane S, Jackson VA, Dahlin CM, Blinderman CD, Jacobsen J, Pirl WF, Billings JA and Lynch TJ: Early palliative care for patients with metastatic non-small-cell lung cancer. N Engl J Med 363: 733-742, 2010.

25. Trotti A, Colevas AD, Setser A, Rusch V, Jaques D, et al: CTCAE v3.0: Development of a comprehensive grading system for the adverse effects of cancer treatment. Semin Radiat Oncol 13: 176-81, 2003. 